\title{
Research of Using Laser Direct Marking Two-Dimensional Car Code Technology on wood
}

\author{
Zhen Wang ${ }^{1,2, a^{*}}$, Aiqun Wang ${ }^{1,2, b}$, Zhuo Ma ${ }^{1,2, c}$ \\ ${ }^{1}$ School of Mechanical Engineering Shandong University, Jinan 250061, P. R. China \\ ${ }^{2}$ Key Laboratory of High-efficiency and Clean Mechanical Manufacture (Shandong University), \\ Ministry of Education, P. R. China \\ awangzhenoo@126.com, bwangaiqun@sdu.edu.cn, ${ }^{\text {c}} 361164029 @ q q . c o m$
}

\begin{abstract}
Keywords: $\mathrm{CO}_{2}$ Laser; Wood Materials; Two-Dimensional Bar Code
Abstract. With the demand and prices for wood products has increased steadily, such as solid wood furniture, wood products material traceability becomes the new requirements. Currently, advanced timber market and the lack of clear and effective regulation of industry standards, the consumer is often misleading consumers. At the same time, because the use of paper and paste, print, etc., wood packaging mark is easy to wear off. In order to solve these problems, we carried out a two-dimensional bar code directly marking a new study using laser marking technology in the wood surface issues. Object of this study is based on our widely distributed larch. We use CO2-S100XP laser to make Data Matrix (DM) two-dimensional bar code on the material surface. We study the feasibility of direct marking dimensional bar code on wood surface. We analyzed the effect of laser parameters on a two-dimensional bar code color and contrast. We use orthogonal experiment to find the optimal combination of laser parameters. This pilot study is the basis of non-metallic laser direct marking, which helps guide the actual marking process.
\end{abstract}

\section{Introduction}

Confusion timber production management system restricts the development of enterprises. Meanwhile timber resource scarcity caused the wood products prices and the proliferation of counterfeit goods. Currently on the market labeled wood products mainly used paper and printing paste, etc., easy to wear off, it caused loss of information. It is not conducive to control information. Take advantage of the two-dimensional bar code permanence and security patch will be applied to two-dimensional digital bar code timber digital management systems and product packaging, so monitor the entire process of production and sales tracking. Two-dimensional code technology can be applied in terms of security and traceability of goods, has been more and more enterprises to accept.

Leone et al. [1] discussed the use of Nd: YAG laser parameters in different timber for removal of. Experimental parameters studied have average power, marking speed and repetition rate, experiments show that the Q-switched diode doubling Nd: YAG laser can be used to process different types of wood, you can get good quality pattern without combustion conditions. Panznera et al. [2] studied wood cutting through laser ablation of the surface, enhanced durability and connectivity gluing surface. The use of excimer lasers, $\mathrm{Nd}$ : YAG lasers and $\mathrm{CO}_{2}$ lasers on different wood experiments. Experimental results show that the laser parameters under certain circumstances can generate no carbonized wood surfaces.

Study abroad focused on marking text or picture messages. The use of laser technology research capacity and safety of the two-dimensional bar code applications on a wooden surface are few. As used herein, the wood surface $\mathrm{CO}_{2}$ laser marking dimensional bar code test, using larch as experimental materials to study the effect of mean power and marking speed laser parameters for contrast. Finally, we get the optimal combination of parameters by experiment. This article has significance for laser direct marking of non-metallic materials.

\section{Equipment, materials and Test design}

Laser marking equipment as used herein can be dynamically focusing the Shenzhen Han's 
$\mathrm{CO}_{2}$-S100XP laser, which produces a wavelength of the laser is $10640 \mathrm{~nm}$ and mean power is $100 \mathrm{w}$, the operating frequency of $1 \sim 0 \mathrm{KHz}$. By adjusting the Q-frequency and Q-release time continuous adjustment of the mean power [3]. Eq. (1) is as follows:

$$
P=Q \text {-requency } \times Q \text {-release time } / 60 \% \times 100
$$

$\mathrm{P}$ is the mean power, $60 \%$ maximum duty cycle for the laser , 100 is a laser maximum power.

In this study, subjects were selected northeast larch forest are the most widely used air-dried wood (equilibrium moisture content of $8.9 \%$, with an average density of $0.598 \mathrm{~g} / \mathrm{cm}^{3}$ ). In order to find the best combination, we mainly analyze barcode quality laser parameters and laser parameters influence.

We based on early experiments with a single variable experimental method to explore the influence of process parameters on processing quality [4]. We got different effects by changing the code marking lasers of various types of $\mathrm{CO}_{2}-\mathrm{S} 100 \mathrm{XP}$ process parameters. The key elements of the pilot study are to select the parameter variables. We discovered mean power (p: w), scanning speed (v: $\mathrm{mm} / \mathrm{s})$, distance between linear patterns $(\mathrm{d}: \mathrm{mm})$ and the defocusing amount $(\Delta \mathrm{f}: \mathrm{mm})$ have big impact on the quality of two-dimensional bar code marking. Use single variable control law, regulating the four parameters; laser parameters study the influence of the contrast of the bar code implementation. The following experiment design scheme based on actual experience in processing:

Experiment 1: mean power (p: w) were taken 6,10, 14, 18, 22, defocusing amount of $-10 \mathrm{~mm}$, scanning speed of $60 \mathrm{~mm} / \mathrm{s}$, distance between linear patterns of $0.2 \mathrm{~mm}$.

Experiment 2: scanning speed (v: $\mathrm{mm} / \mathrm{s})$ were taken $40,60,80,100,120$, mean power values of $10 \mathrm{w}$, defocusing amount of $-10 \mathrm{~mm}$, distance between linear patterns of $0.2 \mathrm{~mm}$.

Experiment 3: distance between linear patterns $(\mathrm{d}: \mathrm{mm})$ were taken $0.1,0.2,0.3,0.4,0.5$, scanning speed of $60 \mathrm{~mm} / \mathrm{s}$, mean power values of $10 \mathrm{w}$, defocusing amount of $-10 \mathrm{~mm}$.

Experiment 4: defocusing amount ( $\Delta \mathrm{f}$ : $\mathrm{mm}$ ) were taken -20,-10,0,10,20, distance between linear patterns of $0.2 \mathrm{~mm}$, scanning speed of $60 \mathrm{~mm} / \mathrm{s}$, mean power values of $10 \mathrm{w}$.

In order to get the optimal parameters, we used the orthogonal experiment to optimize the combination of parameters [5]. Orthogonal selected defocusing amount (A), distance between linear patterns(B), scanning speed(C) and mean power(D),factor of each type taken five levels as shown in Table 1. Contrast index y is orthogonal experiment.

Table 1 Factor levels table

\begin{tabular}{cccccc}
\hline \multirow{2}{*}{ factor } & \multicolumn{5}{c}{ level } \\
& 1 & 2 & 3 & 4 & 5 \\
\hline $\mathrm{A}(\mathrm{mm})$ & -20 & -10 & 0 & 10 & 20 \\
$\mathrm{~B}(\mathrm{~mm})$ & 0.1 & 0.2 & 0.3 & 0.4 & 0.5 \\
$\mathrm{C}(\mathrm{mm} / \mathrm{s})$ & 40 & 60 & 80 & 100 & 120 \\
$\mathrm{D}(\mathrm{w})$ & 6 & 10 & 14 & 18 & 22 \\
\hline
\end{tabular}

We used Sirius USB camera acquisition of two-dimensional bar code after engraving, then use the DVT intellect 1.5.0 software to read sampled two-dimensional barcode image and according to ISO-IEC-16022(AIM) grade standards [6], based on the contrast as evaluation index of barcode image quality. Barcode is established by article and empty light reflectivity of different to read, different colors of light have different reflectivity, which leads to the change of the contrast.

\section{Experiment and result analysis}

When the wood surface is irradiated with a laser, the laser irradiation part slight carbonation phenomenon occurs with non-irradiated portion so as to form a clear color difference marked effect. Changes in laser parameters will change in the energy density of the wood surface [7], with the decrease or increase of laser energy density, laser engraved wood surface in standard will be a different situation. Figure. 1 can be seen when small energy density, the smaller the color change marking the area as shown in Figure.1 (a); as the energy density increases watch engraved marker 
region to achieve a better effect shown in figure.1 (b); when energy density is too large when it will occur serious carbonized color background area also changes, resulting in smaller contrast cannot even read, as shown figure.1(c).

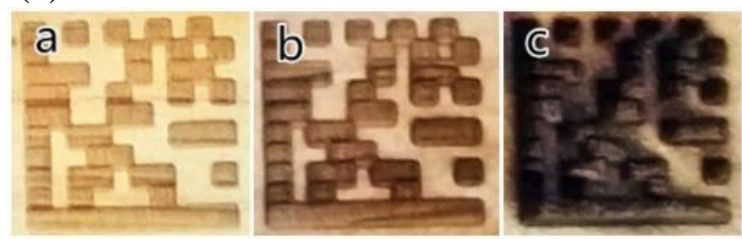

Fig.1. Barcode at different energy densities

It was found that when the mean power is less than $4.00 \mathrm{w}$, laser failed to have an impact on the material surface, table engraved bar code is not visible. We gradually increase the average power was found two-dimensional bar gradually clear, until you get a clear contrast. When the mean power exceeds $20.00 \mathrm{w}$ the wood surface occur serious carbonization. Bar code cannot be read. Fig.2 (a) contrast curve analysis found that the contrast will begin with the mean power becomes larger barcode and gradually increase the mean power reaches a certain value, the surface will be marking severe carbonation occurs, decrease the contrast value.

Scanning speed determines the distance between adjacent laser points, the smaller the distance, the slower the rate of adjacent laser point. In order to obtain the same contrast, in the case of high speed is necessary that we should reduce the average power corresponding. We analysis Fig.2 (b) contrast curve found that the scanning speed of less than $40 \mathrm{~mm} / \mathrm{s}$ when the contrast is low, which is due to the accumulation of the laser beam energy so that was marking the surface temperature carbonization phenomenon occurs more serious cause. With increasing velocity contrast will become larger when the speed exceeds $60 \mathrm{~mm} / \mathrm{s}$ continues to increase, the contrast will gradually be reduced, the occurrence of this situation analysis found that when certain mean power, marking experiment with speed It accelerates, laser engraved with the table area without adequate duration of action, leading to decrease in contrast.

Distance between linear patterns is too small, it will also cause the accumulation of laser energy and decreased contrast. Fig.2 (c) contrast curve analysis found that when the wire spacing becomes larger, get a higher contrast value. With more than $0.2 \mathrm{~mm}$ to continue to increase the contrast, it found that contrast sharply reduced, found the distance between linear patterns is too large, the contact area of laser marking and material surface becomes smaller, namely to reduce the amount of material vaporized, causing a decrease in contrast.

Geometrical optics theory suggests that when the positive and negative mark from the focal plane and the plane distance equal to the corresponding power density approximately the same plane. We analysis Fig.2 (d) contrast curve found that the effect of marking is actually obtained and different. For wood materials, negative defocus effect will produce better contrast. 


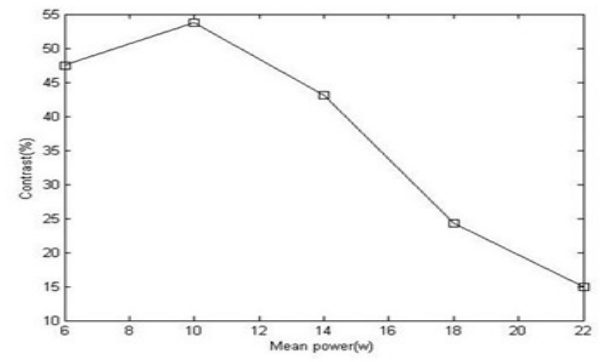

(a) Mean power

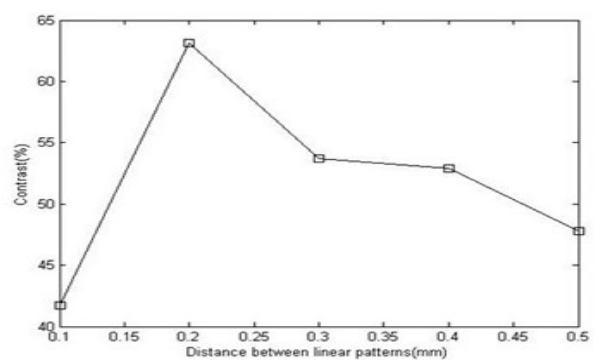

(c) Distance between linear patterns

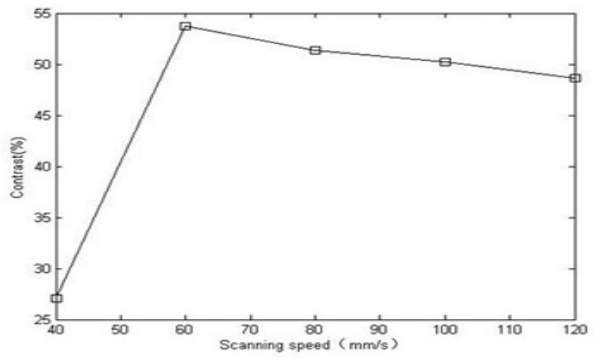

(b) Scanning speed

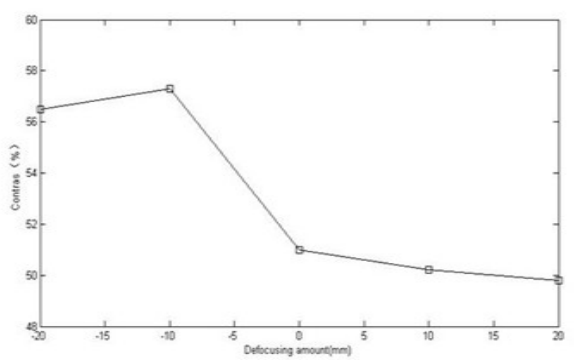

(d) Defocusing amount

Fig.2.The influence of different laser parameters on the contrast curve

\section{Laser combination parameters optimization}

Laser parameters are optimized for the laser is tunable and physical properties of the material to be processed to optimize the analysis process. In this process, the mark is a measure of the quality of the merits of the standard process parameters, while the guidance of feedback regulation of process parameters. In orthogonal test, factor A test conducted five times, and in these five trials, five levels of factor B, C, D are also each conducted a test, all tests will be divided into five groups, then this differences among the five sets of data on five levels reflect differences in factor A. factor Different average level is shown in figure .3 .

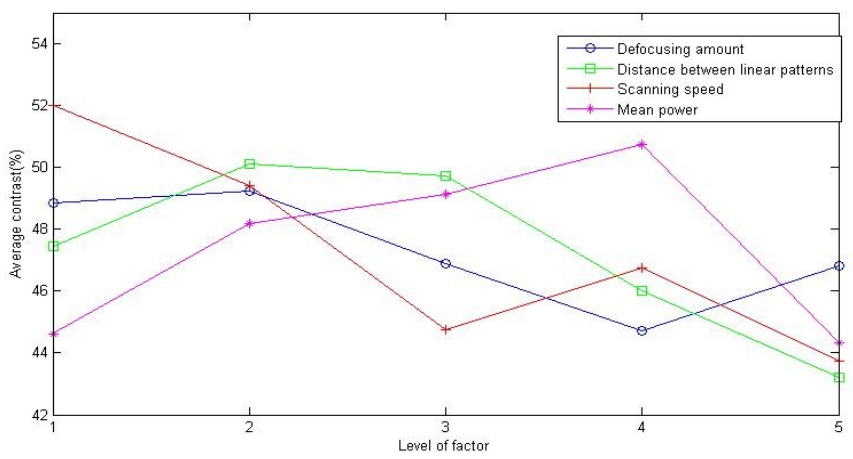

Fig.3.The average contrast each factor level

Within the five levels of the experiment, analysis Fig. 3 available:

$$
\begin{array}{cc}
A_{2}>A_{1}>A_{3}>A_{5}>A_{4} & B_{2}>B_{3}>B_{1}>B_{4}>B_{5} \\
C_{1}>C_{5}>C_{2}>C_{4}>C_{3} & D_{4}>D_{3}>D_{2}>D_{1}>D_{5}
\end{array}
$$

In the range of the parameters, we can get the larch surface on the optimal parameter 
combination $A_{2} B_{2} C_{1} D_{4}$ : defocusing amount of - $10 \mathrm{~mm}$, distance between linear patterns of $0.2 \mathrm{~mm}$, scanning speed of $40 \mathrm{~mm} / \mathrm{s}$, mean power of $18 \mathrm{w}$, with the result of the experiment verification, test requirements in line with expectations.

\section{Conclusion}

In this paper, we did a lot of tests in larch surface using $\mathrm{CO}_{2}-\mathrm{S} 100 \mathrm{XP}$ laser. By analyzing the color of the bar code and bar code contrast, it proved the feasibility of marking a two-dimensional bar code laser directly at the wood surface. Laser power $10.00 \mathrm{w} \sim 18.00 \mathrm{w}$ and marking speed within $40 \mathrm{~mm} / \mathrm{s} \sim 80 \mathrm{~mm} / \mathrm{s}$ range, does not appear serious carbonation phenomenon, literacy rate is better. Within the range of experimental parameters, laser power and laser marking speed barcode contrast influence is more evident. When the laser power is less than $4.00 \mathrm{w}$ or marking speeds greater than $120 \mathrm{~mm} / \mathrm{s}$, it will not form a significant mark in larch surface. Increasing the laser power or reduce marking speed table will be a significant moment carbonization area will occur. Laser marking in the focal plane mark not get the desired effect for wood. When negative defocus slight carbonation phenomenon occurs, increasing the contrast of the bar code. Line spacing between $0.1 \mathrm{~mm} \sim 0.3 \mathrm{~mm}$ marking effect is better.

\section{Acknowledgement}

This work was financially supported by foundation item of promotive research fund for excellent young and middle-aged scientists of Shandong Province [item number: BS2012zz005].

\section{References}

[1] C. Leone, V, Lopresto. Wood engraving by Q-switched diode-pumped frequency-doubled Nd: YAG green laser [J]. Optics and Lasers in Engineering 47 (2009) 161-168.

[2] Panzner M, Wiedemann G, Henneberg K, et al. Experimental investigation of the laser ablation process on wood surfaces [J]. Applied Surface Science, 1998, 127:787-792.

[3] Laser marking technology handbook [M]. Shenzhen: laser technology co., LTD, 2009.

[4] Zhuo Ma, Jianmei Li, Changhou Lu. The laser directly on the two dimensional barcode technology in banana on the surface of the applied research [J]. Applied laser, 2015, 01 period (1).

[5] Yanhong Fan, Yuchegn Zhou, Yake Li. Orthogonal experimental method in the application of laser cutting parameters selection [J]. Journal of laser, 2008, 6 (6): 83-84.

[6] ISO/IEC 16022:2000. Information technology-International symbology specification-Data matrix. 2004.

[7] Yousheng Li, Xiao Xi, Jianshe Lei. Best tuning Q laser engraved scale parameter study [J]. Journal of optoelectronics, lasers, 1998, 2:152-154. 